\title{
SARS-CoV-2 infection and COVID-19 vaccination rates in pregnant women in Scotland
}

\author{
Sarah J. Stock ${ }^{1,2}{ }^{\star}$, Jade Carruthers ${ }^{2}$, Clara Calvert ${ }^{1}$, Cheryl Denny ${ }^{\circledR 2}$, Jack Donaghy ${ }^{2}$, \\ Anna Goulding ${ }^{102}$, Lisa E. M. Hopcroft ${ }^{2,3}$, Leanne Hopkins², Terry McLaughlin'2, Jiafeng Pan², \\ Ting Shi ${ }^{1}{ }^{1}$, Bob Taylor ${ }^{2}{ }^{2}$, Utkarsh Agrawal ${ }^{4}$, Bonnie Auyeung ${ }^{5}$, Srinivasa Vittal Katikireddi ${ }^{2,6}$, \\ Colin McCowan ${ }^{4,7}$, Josie Murray ${ }^{2}$, Colin R. Simpson ${ }^{11,8}{ }^{1}$, Chris Robertson ${ }^{2,9}$, Eleftheria Vasileiou', \\ Aziz Sheikh ${ }^{1}{ }^{1}$ and Rachael Wood ${ }^{1,2}$
}

\begin{abstract}
Population-level data on COVID-19 vaccine uptake in pregnancy and SARS-CoV-2 infection outcomes are lacking. We describe COVID-19 vaccine uptake and SARS-CoV-2 infection in pregnant women in Scotland, using whole-population data from a national, prospective cohort. Between the start of a COVID-19 vaccine program in Scotland, on 8 December 2020 and 31 October 2021, 25,917 COVID-19 vaccinations were given to 18,457 pregnant women. Vaccine coverage was substantially lower in pregnant women than in the general female population of 18-44 years; 32.3\% of women giving birth in October 2021 had two doses of vaccine compared to $77.4 \%$ in all women. The extended perinatal mortality rate for women who gave birth within $28 \mathrm{~d}$ of a COVID-19 diagnosis was 22.6 per 1,000 births (95\% CI 12.9-38.5; pandemic background rate 5.6 per 1,000 births; 452 out of 80,$456 ; 95 \%$ Cl 5.1-6.2). Overall, $77.4 \%$ (3,833 out of 4,950; $95 \%$ Cl 76.2-78.6) of SARS-CoV-2 infections, $90.9 \%$ (748 out of 823; 95\% CI 88.7-92.7) of SARS-CoV-2 associated with hospital admission and $98 \%$ (102 out of 104; $95 \%$ CI 92.5-99.7) of SARS-CoV-2 associated with critical care admission, as well as all baby deaths, occurred in pregnant women who were unvaccinated at the time of COVID-19 diagnosis. Addressing low vaccine uptake rates in pregnant women is imperative to protect the health of women and babies in the ongoing pandemic.
\end{abstract}

C omprehensive whole-population data on COVID-19 vaccine uptake in pregnancy and SARS-CoV-2 infection rates and COVID-19 outcomes are lacking ${ }^{1}$. Such data are required to help guide decision making by women, clinicians and policymakers on measures to prevent and control COVID-19 in pregnancy, in particular, vaccination.

The first case of SARS-CoV-2 in Scotland was identified on 1 March 2020. Different SARS-CoV-2 variants have predominated in subsequent waves of infection, with wild type predominant initially, the Alpha variant dominant from January 2021 and the Delta variant dominant from May 2021. SARS-CoV-2 testing was initially restricted due to limited availability, with availability in the community for any symptomatic adult available from 18 May 2020. Although not uniformly implemented in all maternity units, routine SARS-CoV-2 testing of maternity admissions was instituted from 1 December 2020.

Pregnant women do not seem to be more susceptible to SARS-CoV-2 infection than non-pregnant women, but they are at higher risk of severe COVID-19 disease ${ }^{2-4}$. Compared to non-pregnant women of reproductive age, pregnant women with SARS-CoV-2 infection are more likely to be admitted to critical care, receive invasive ventilation and extracorporeal membrane oxygenation and $\mathrm{die}^{2,3}$. COVID-19 in pregnancy is associated with increased risk of the pregnancy specific complications pre-eclampsia, preterm birth and stillbirth ${ }^{2,5-10}$.
Despite widespread recognition of potential vulnerability to COVID-19, pregnant women were excluded from pre-marketing clinical trials studying COVID-19 vaccines ${ }^{11,12}$. As a result, evidence to inform decision making on vaccination was largely absent when vaccination programs started and recommendations on vaccination in pregnancy have varied over time as well as by country ${ }^{13}$. Post-marketing surveillance data suggest that COVID-19 vaccine effectiveness is broadly similar to that in non-pregnant individuals ${ }^{14,15}$. Data on the safety of vaccines in pregnancy comes from preclinical studies of COVID-19 vaccines in animals ${ }^{16}$, findings from women who had unanticipated pregnancies while participating in clinical tri$\mathrm{als}^{17}$ and accumulating pharmacovigilance data ${ }^{18-21}$, all of which are reassuring regarding COVID-19 vaccine safety in pregnancy.

The COVID-19 vaccination program in Scotland commenced on 8 December 2020 and policies on the provision of COVID-19 vaccination to pregnant women evolved over time (Fig. 1). From 16 April 2021 the recommendation has been that women who are pregnant should be offered vaccination at the same time as non-pregnant women, based on their age and clinical risk group and no pregnancy specific pre-vaccination counseling is required.

The COVID-19 in Pregnancy in Scotland (COPS) study (a sub-study of EAVE II (Early Pandemic Evaluation and Enhanced Surveillance of COVID-19) ${ }^{22,23}$ ) is a national, prospective dynamic cohort of all women who were pregnant on, or became pregnant after, 1 March 2020; linked to SARS-CoV-2 infection data and

'University of Edinburgh Usher Institute, Edinburgh, UK. ${ }^{2}$ Public Health Scotland, Scotland, UK. ${ }^{3}$ The DataLab, Nuffield Department of Primary Care Health Sciences, University of Oxford, Oxford, UK. ${ }^{4}$ School of Medicine, University of St Andrews, St Andrews, UK. ${ }^{5}$ School of Philosophy, Psychology and Language Sciences, University of Edinburgh, Edinburgh, UK. ${ }^{6} \mathrm{MRC} / \mathrm{CSO}$ Social \& Public Health Sciences Unit, University of Glasgow, Glasgow, UK. ${ }^{7}$ Institute of Health \& Wellbeing, University of Glasgow, Glasgow, UK. ${ }^{8}$ School of Health, Wellington Faculty of Health, Victoria University of Wellington, Wellington, New Zealand. ${ }^{9}$ Department of Mathematics and Statistics, University of Strathclyde, Glasgow, UK. 凶e-mail: sarah.stock@ed.ac.uk 
Cohorts invited for vaccination

Frontline health and social care workers

Carers in care homes

Clinically extremely vulnerable

\begin{tabular}{c}
\hline All 50-54 years old \\
\hline All 40-49 years old \\
All 30-39 years old
\end{tabular}

All 18-29 years old

At risk $12-15$ years old

All $16-17$ years old

All 12-15 years old

Boosters: frontline health and socia care workers

Carers in care homes Clinically extremely vulnerable
2020

DEC.

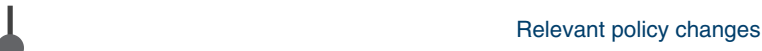

08 Vaccination program started

30 Clarification that pregnant women in priority risk groups could be offered vaccine

JAN

FEB

22 National pathway for vaccination of pregnant women in priority groups established

- Information leaflets for pregnant women produced

09 Women with gestational diabetes added to the 'at-risk' group offered vaccination

APR

7 Preference for alternative to Oxford-AstraZeneca AZD1222 vaccine in those aged $<30$ years announced

16 Pregnant women in any priority group could be offered vaccination

MAY

- No specific prevaccination counselling required

- Preference for Pfizer-BioNTech or Moderna vaccines where available for all pregnant women regardless of age

JUN

\section{JUL}



$$
A \cup G
$$

UUG.

01 Those with severe immunosuppression offered a third dose of vaccination as part of primary course

14 Booster vaccinations for those $>6$ months since second dose

OCT

-

NOV

Current policy Women who are pregnant

should be offered vaccination at the same time

2021

as non-pregnant women based on their age

and clinical risk group.

Fig. 1 | Populations invited for COVID-19 vaccination in Scotland over time. Summary of population cohorts invited for COVID-19 vaccination over time in Scotland from the start of the vaccination program on 8 December 2020 (left) and policy changes relevant to vaccination in pregnant women (right).

COVID-19 vaccination data ${ }^{24,25}$. The aims of this study were to use data from the COPS cohort to describe the uptake and coverage of COVID-19 vaccination in pregnant women; to describe the incidence of SARS-CoV-2 infection in pregnant women; to describe rates of hospital admission, critical care admission, preterm birth and extended perinatal mortality following SARS-CoV-2 infection in pregnancy; and to explore the effect of COVID-19 vaccination status on these post-infection outcomes.

\section{Results}

Cohort. We used the COPS database linked to records of COVID19 vaccinations delivered and SARS-CoV-2 infections diagnosed up 


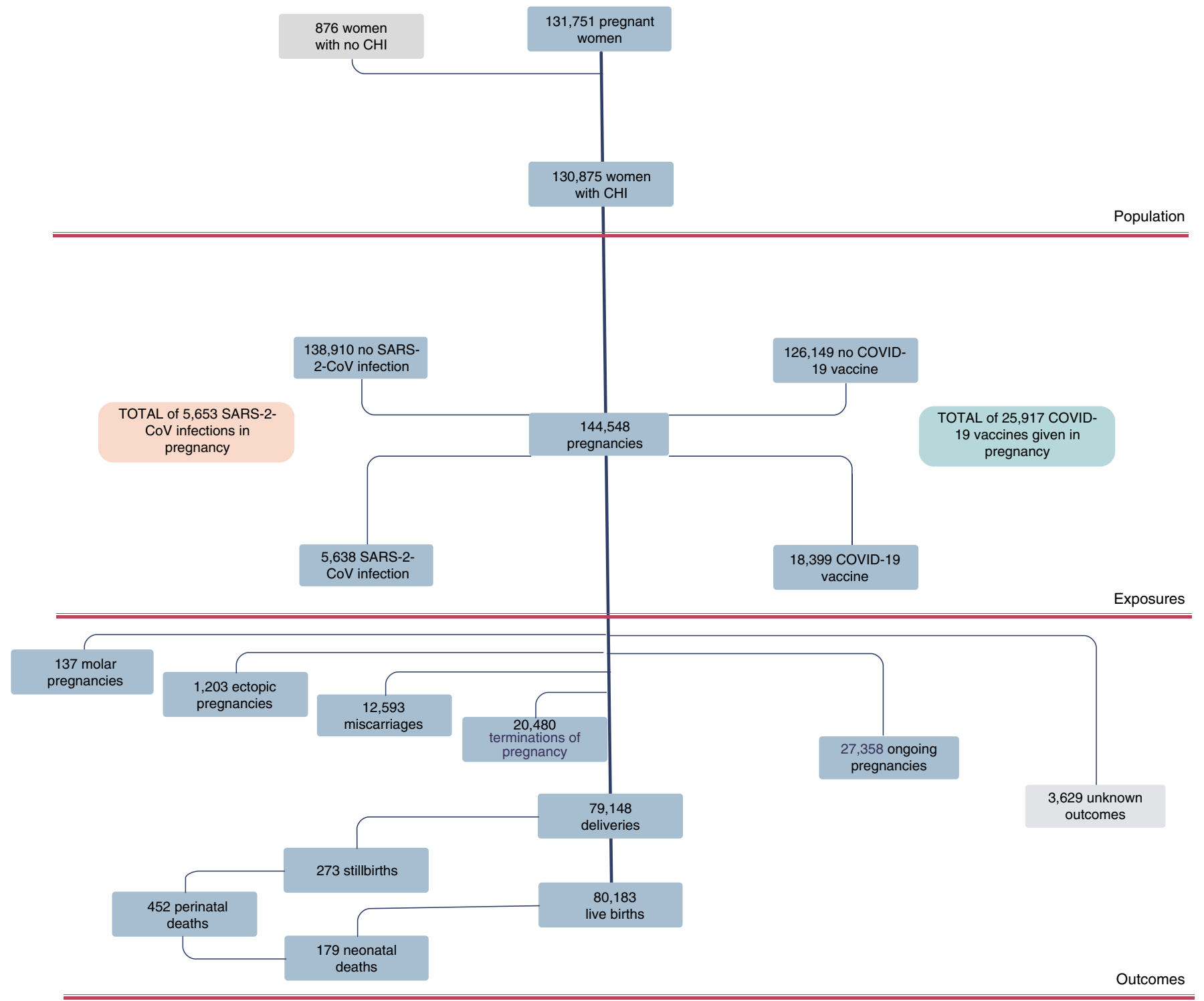

Fig. 2 | Summary of participants in the COVID-19 in Pregnancy in Scotland cohort. Flow chart summarizing participants in the COPS cohort. Counts for molar pregnancies, ectopic pregnancies, miscarriages, terminations and deliveries are numbers of pregnancies. Live births, stillbirths and neonatal deaths are numbers of babies.

to and including 31 October 2021. This included data on a total of 145,424 pregnancies in 131,751 women. Overall, $144,548(99.4 \%)$ of these pregnancy records (and 130,875, 99.3\% of the women) had an associated unique community health index (CHI) number used for data linkage. Pregnancy records with no associated $\mathrm{CHI}$ number are likely to relate to duplicate records so were excluded from this analysis. Figure 2 describes the participants in the cohort. Overall, 117,190 pregnancies were complete, with 13,933 ending in early pregnancy loss (miscarriage, molar or ectopic pregnancy), 20,480 resulting in termination of pregnancy, 79,148 resulting in delivery and 3,629 having an unknown outcome. The deliveries resulted in 273 stillbirths and 80,183 live births, of which 179 resulted in neonatal death. Overall, 27,358 pregnancies were ongoing on 31 October 2021.

Pregnancy data were linked with national data on confirmed SARS-CoV-2 infection and COVID-19 vaccination using CHI number. Overall, 99\% of all the PCR with reverse transcription (RTPCR) samples within the national testing database since the start of the pandemic, and $99.8 \%$ of all records for vaccinations given from the start of the program, have an associated $\mathrm{CHI}$ number.
Infection and vaccination in pregnancy was defined as infection diagnosed or vaccination given at any point from the date of conception $(2+0$ weeks gestation) to the date the pregnancy ends inclusive. The date of first positive viral RT-PCR sample collection was taken as the date of onset of the first episode of COVID-19. Subsequent episodes were recorded if a positive viral RT-PCR sample was taken $\geq 90 \mathrm{~d}$ after a first positive sample.

To explore the impact of vaccination status on SARS-CoV-2 infection, women were grouped as follows: unvaccinated (no previous COVID-19 vaccination before the date of onset of COVID19 or with one dose of vaccination $\leq 21 \mathrm{~d}$ before the date of onset); partially vaccinated (one dose of vaccination $>21 \mathrm{~d}$ before the date of onset of COVID-19 or two doses of vaccination with the second dose administered $\leq 14 \mathrm{~d}$ before the date of onset); or fully vaccinated (two doses of vaccination with the second dose $>14 \mathrm{~d}$ before the date of onset of COVID-19). We also report the number of third or booster doses given, but have not included these in definitions of vaccination status, as third and booster doses in Scotland were only recommended from 14 and 20 September 2021, respectively. 

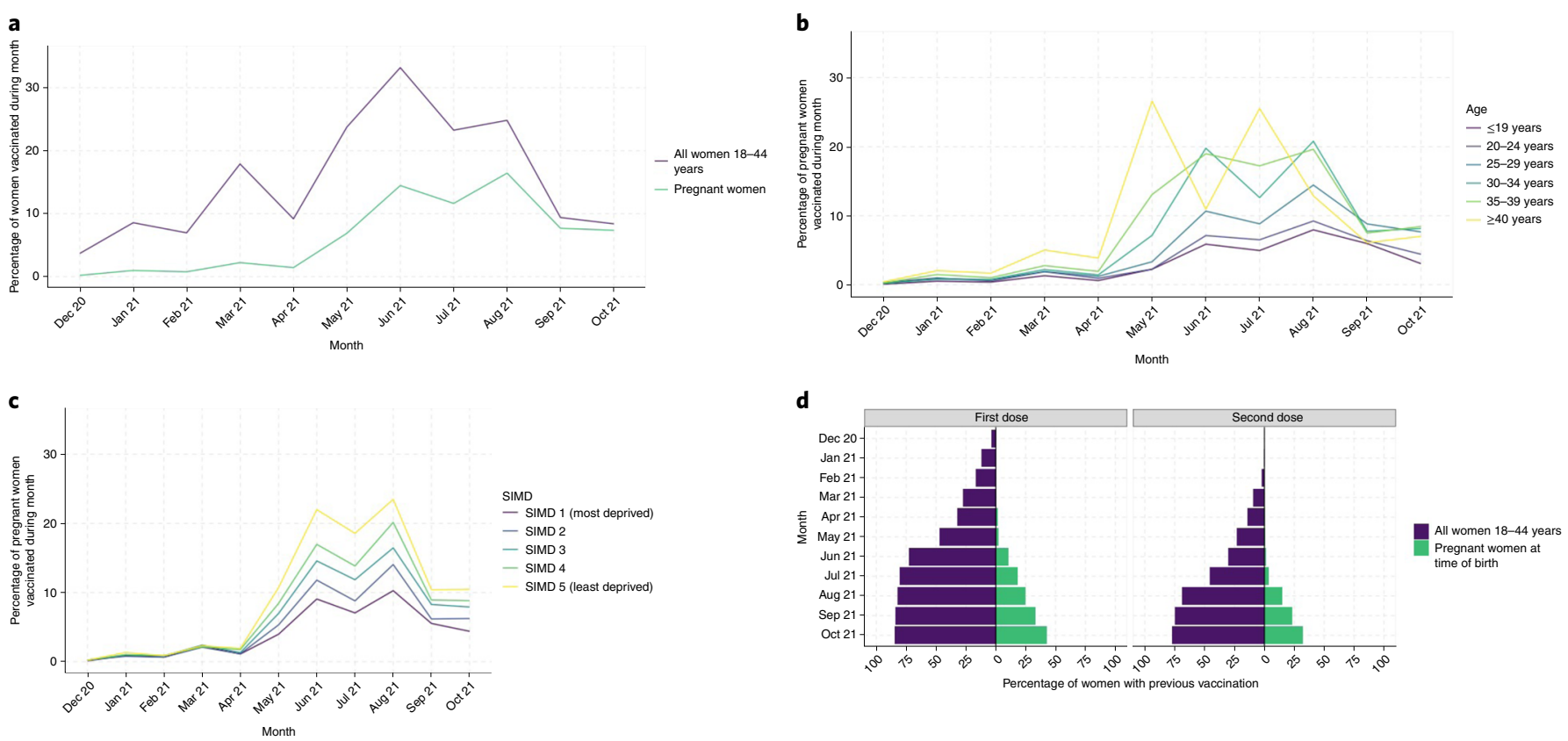

d

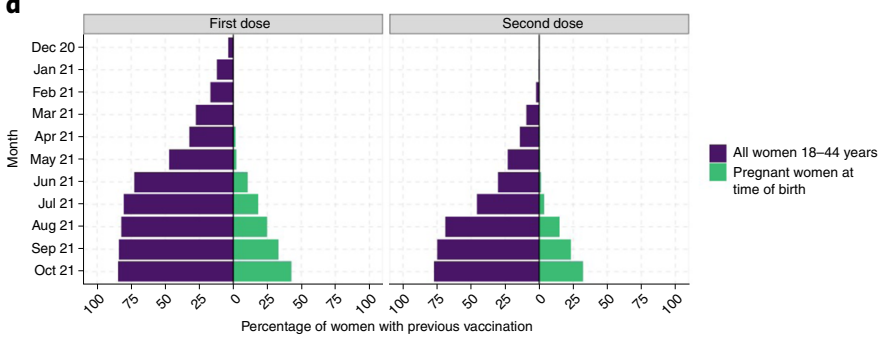

Fig. 3 | COVID-19 vaccination uptake and coverage. a, Monthly uptake of COVID-19 vaccination in all women 18-44 years and in pregnant women. b, Monthly uptake of COVID-19 vaccination in pregnant women by age group. c, Monthly uptake of COVID-19 vaccination in pregnant women by SIMD quintile. SIMD, Scottish Index Multiple Deprivation, with SIMD 5 being least deprived and 1 being most deprived. d, Vaccine coverage in the general female population in women age 18-44 years (purple) and percentage of pregnant women vaccinated before the time of birth (green). Numbers of women $18-44$ years were derived from 2020 mid-year population estimates ${ }^{26}$.

COVID-19 vaccine coverage and uptake in pregnancy. From 1 December 2020 to 31 October 2021 a total of 25,917 COVID-19 vaccinations (12,518 (48\%) first doses, 12,194 (47\%) second doses and $1,205(5 \%)$ third or booster doses) had been given in 18,399 pregnancies. A total of 9,905 (38.2\%; 95\% CI 37.6-38.8) vaccinations were given in the first trimester of pregnancy (at $2+0$ to $13+6$ weeks gestation); 9,317 (35.9\%; 95\% CI 35.4-36.5) in the second trimester (at $14+0$ to $27+6$ weeks gestation); and 6,695 $(25.8 \% ; 95 \%$ CI 25.3-26.4) in the third trimester (at $28+0$ weeks gestation or over). Of the vaccinations given, 20,572 (79.4\%; 95\% CI 78.9-79.9) were Pfizer-BioNTech BNT162b2 messenger RNA vaccine; 3,224 (12.4\%; 95\% CI 12.0-12.8) were Moderna mRNA-1273 mRNA vaccine; and 2,121 (8.2\%; 95\% CI 7.9-8.5) were Oxford-AstraZeneca AZD1222 viral vector vaccine (given mainly early in the vaccine program to pregnant women with a clinical risk factor indicating vaccination).

We used two different metrics to describe vaccination in pregnancy, monthly uptake in women who were pregnant during the month and coverage (the proportion of women who, at the time of giving birth, had been vaccinated, whether vaccination was before or during pregnancy). Uptake and coverage data in the general female population of reproductive age (18-44 years) were provided for context, generated from data collected by Public Health Scotland ${ }^{26}$.

COVID-19 vaccine uptake among pregnant women each month was consistently lower than that in the general female population of reproductive age (Fig. 3a). Vaccine uptake was consistently lowest in younger ( $\leq 20$ years) pregnant women and those living in the most deprived areas of Scotland (Fig. 3b,c).

COVID-19 vaccine coverage increased over time, but has consistently been substantially lower among pregnant women than in the general female population of reproductive age (Fig. 3d). In October $2021,4,064$ women gave birth, of whom 1,738 (42.8\%; 95\% CI 41.2-44.3) had received any COVID-19 vaccination before delivery, with $1,311(32.3 \%$; $95 \%$ CI $30.8-33.7)$ of the women having received two primary doses of vaccination and 36 women $(0.9 \%$;
95\% CI 0.6-1.2) having received a third or booster dose of vaccination. In contrast, by 31 October $2021,84.7 \%$ (803,241 out of 947,$984 ; 95 \%$ CI 84.7-84.8) of women aged $18-44$ years in the general population had received any vaccination, $77.4 \%$ had received two doses $(733,942$ out of 947,$984 ; 95 \%$ CI $77.3-77.5)$ and $7.0 \%$ had received a third dose or booster dose $(66,001$ out of 947,$984 ; 95 \%$ CI 6.9-7.0).

SARS-CoV-2 outcomes in vaccinated and unvaccinated pregnant women. Between 1 March 2020 and 31 October 2021, there were 5,653 confirmed SARS-CoV-2 infections in pregnancy. Overall, rates of SARS-CoV-2 infection in pregnancy showed similar patterns to those in the general female population of reproductive age, with peaks of infection in October 2020, January 2021 and September 2021 (Fig. 4a). SARS-CoV-2 infection rates have been consistently highest in pregnant women living in the most deprived areas and in younger, compared to older, pregnant women (Fig. 4b,c).

Early in the pandemic, it is highly likely that there was under-ascertainment of cases of SARS-CoV-2 infection due to limited test availability and testing capacity (Fig. 4a). Restricting the COPS cohort to only data from 1 December 2020 onward (the date that routine SARS-CoV-2 testing was recommended for all admissions to maternity care and the COVID-19 vaccination program started in Scotland) reduces the size of the cohort, but allows more comparable estimation of rates of severe outcomes (hospital admission, critical care admission and perinatal mortality) associated with SARS-CoV-2 infection in vaccinated and unvaccinated women. From 1 December 2020 onward, the COPS database included linked data on a total of 91,183 pregnancies in 87,694 women.

There were 4,950 confirmed SARS-CoV-2 infections in pregnancy from 1 December 2020. SARS-CoV-2 infections were relatively evenly spread throughout pregnancy $(1,543$ (31.2\%; $95 \% \mathrm{CI}$ 29.9-32.5) diagnosed in the first trimester of pregnancy; 1,850 (37.4\%; 95\% CI 36.0-38.7) in the second trimester; and 1,557 (31.5\%; 95\% CI 30.2-32.8) in the third trimester). 

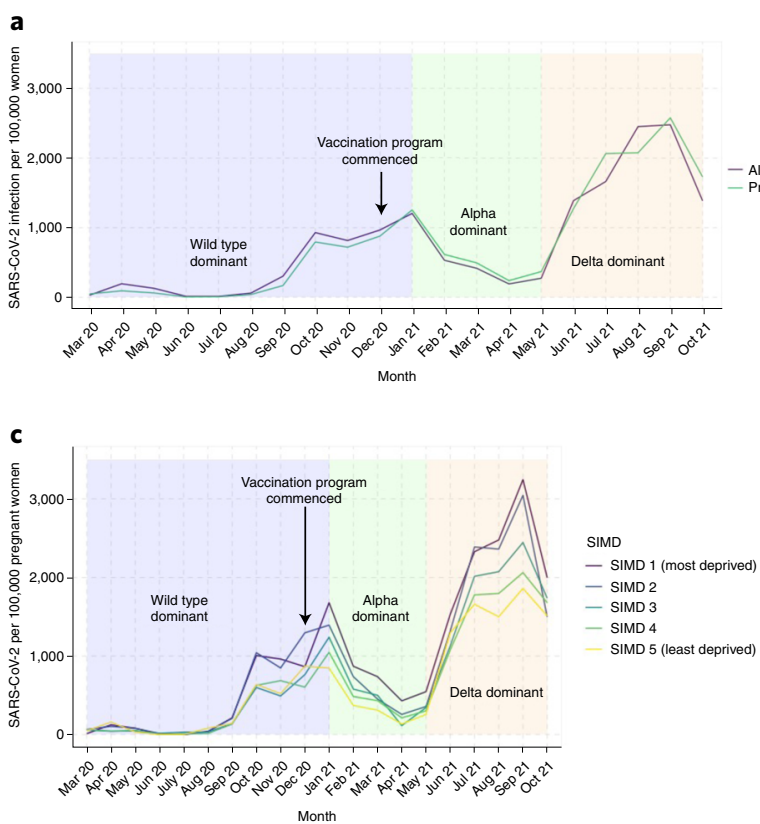

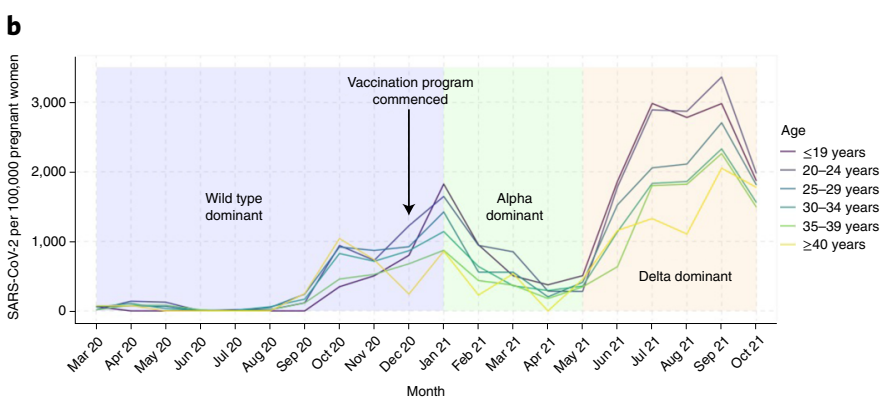

d

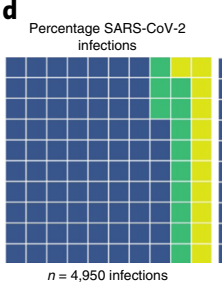

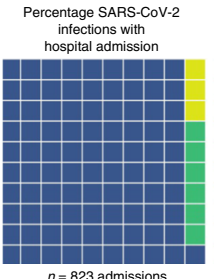

$n=823$ admissions

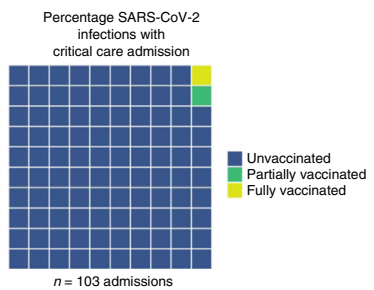

Fig. 4 | SARS-CoV-2 in pregnancy and outcomes by vaccination status. a, SARS-CoV-2 in all women 18-44 years per 100,000 women and in pregnant women. b, Monthly rates of SARS-CoV-2 per 100,000 pregnant women by age group c, Monthly rates of SARS-CoV-2 per 100,000 pregnant women by SIMD. d, Percentage of cases of SARS-CoV-2 infection in pregnancy occurring 1 December 2020 to 31 October 2021 inclusive, cases with associated hospital admission and cases with associated critical care admission, by vaccination status at the date of onset of COVID-19. Unvaccinated is defined as no COVID-19 vaccination before the date of onset of COVID-19 or one dose of vaccination $\leq 21 \mathrm{~d}$ previously in line with standard definitions used by Public Health Scotland.

Overall, 823 of the 4,950 SARS-CoV-2 infections in pregnancy from 1 December 2020 onward (16.6\%; 95\% CI 15.6-17.7) were associated with any hospital admission (date of onset of infection occurred during a hospital admission or within $14 \mathrm{~d}$ before admission) and 104 SARS-CoV-2 infections in pregnancy $(2.1 \%$; $95 \% \mathrm{CI}$ 1.7-2.6) were associated with a critical care admission (date of onset of infection occurred during a critical care admission or within $21 \mathrm{~d}$ before admission). In first trimester SARS-CoV-2 infections, $6.7 \%$ (103 out of 1,$543 ; 95 \%$ CI 5.5-8.1) were associated with any hospital admission, compared to $10.7 \%$ (198 out of 1,850 cases; $95 \%$ CI 9.3-12.2) of those in the second trimester and 33.5\% (522 out of 1,$557 ; 95 \%$ CI 31.2-35.9) of those in the third trimester. No (0 out of 1,543 cases; $95 \%$ CI $0-0.3$ ) SARS-CoV-2 infections in the first trimester were associated with critical care admission, compared to $2.0 \%$ (37 out of 1,850 cases; $95 \%$ CI $1.4-2.8$ ) of those in the second trimester and $4.3 \%$ (67 out of 1,$557 ; 95 \%$ CI 3.4-5.5) of those in the third trimester.

From 1 December 2020 onward, 77.4\% of SARS-CoV-2 infections in pregnancy $(3,833$ out of 4,$950 ; 95 \%$ CI $76.2-78.6)$ occurred in women who were unvaccinated at the date of onset of infection, with $11.5 \%$ (567 out of 4,950 ; $95 \%$ CI $10.6-12.4$ ) in partially vaccinated women and $11.1 \%$ (550 out of 4,950 ; $95 \%$ CI $10.3-12.0$ ) in fully vaccinated women.

Of the SARS-CoV-2 infections in pregnancy that occurred in unvaccinated women, $19.5 \%$ (748 out of 3,833; 95\% CI 18.3-20.8) were associated with hospital admission, compared to $8.3 \%$ (47 out of 567; 95\% CI $6.2-10.9$ ) of those in partially vaccinated women and $5.1 \%$ ( 28 out of $550 ; 95 \%$ CI $3.5-7.4$ ) of those in fully vaccinated women. A total of $2.7 \%$ (102 out of 3,833 ; 95\% CI 2.2-3.2) of the SARS-CoV-2 infections in pregnancy that occurred in unvaccinated women were associated with a critical care admission, compared to $0.2 \%$ ( 1 out of $567 ; 95 \%$ CI $0.01-1.1$ ) of those in partially vaccinated women and $0.2 \%$ ( 1 out of 550 cases; $95 \%$ CI $0.01-1.2$ ) of those in fully vaccinated women. This means that while $77.4 \%$ (3,833 out of
4,950; 95\% CI 76.2-78.6) of SARS-CoV-2 infections in pregnancy occurred in unvaccinated women, $90.9 \%$ (748 out of 823 ; $95 \%$ CI 88.7-92.7) of infections associated with hospital admission and 98.1\% (102 out of 104; 95\% CI 92.5-99.7) of infections associated with critical care admission, were in unvaccinated women (Fig. $4 \mathrm{~d}$ ). To date, there has been one maternal death following SARS-CoV-2 infection in pregnancy in Scotland.

A total of 2,364 babies have been born to women who had SARS-CoV-2 infection in pregnancy between 1 December 2020 and 31 October 2021. Of these, 2,353 were live births, of which 241 were preterm births ( $<37$ weeks gestation; preterm birth rate $10.2 \%$; $95 \%$ CI 9.1-11.6). Overall, 610 of the live births and 101 of the preterm births occurred within $28 \mathrm{~d}$ of the date of onset of the mother's SARS-CoV-2 infection, giving a preterm birth rate among babies born within $28 \mathrm{~d}$ of SARS-CoV-2 infection of $16.6 \%$ (95\% CI 13.7-19.8).

Of the 2,364 total births, 11 were stillbirths (deaths in utero $\geq 24$ weeks gestation) and eight live births resulted in neonatal deaths (death within $28 \mathrm{~d}$ of birth), giving an extended perinatal mortality rate of 8.0 per 1,000 births following SARS-CoV-2 infection at any point in pregnancy (19 out of 2,$364 ; 95 \%$ CI $5.0-12.8$ ). Ten of the stillbirths and four neonatal deaths occurred in babies born within $28 \mathrm{~d}$ of the onset of maternal infection, giving an extended perinatal mortality rate of 22.6 per 1,000 births (14 out of 620 , $95 \% 12.9-38.5)$ in this population. All perinatal deaths following SARS-CoV-2 infection in pregnancy occurred in women who were unvaccinated at the time of SARS-CoV-2 infection. We do not have access to detailed clinical records to assess whether COVID-19 directly or indirectly contributed to the preterm births and deaths seen following maternal infection.

For comparison, the background preterm birth rate during the pandemic (from 1 March 2020 to 31 October 2021) was $8.0 \%(6,381$ out of 80,183 live births; $95 \%$ CI $7.8-8.1)$ and the extended perinatal mortality rate was 5.6 per 1,000 births ( 452 out of 80,456 total 


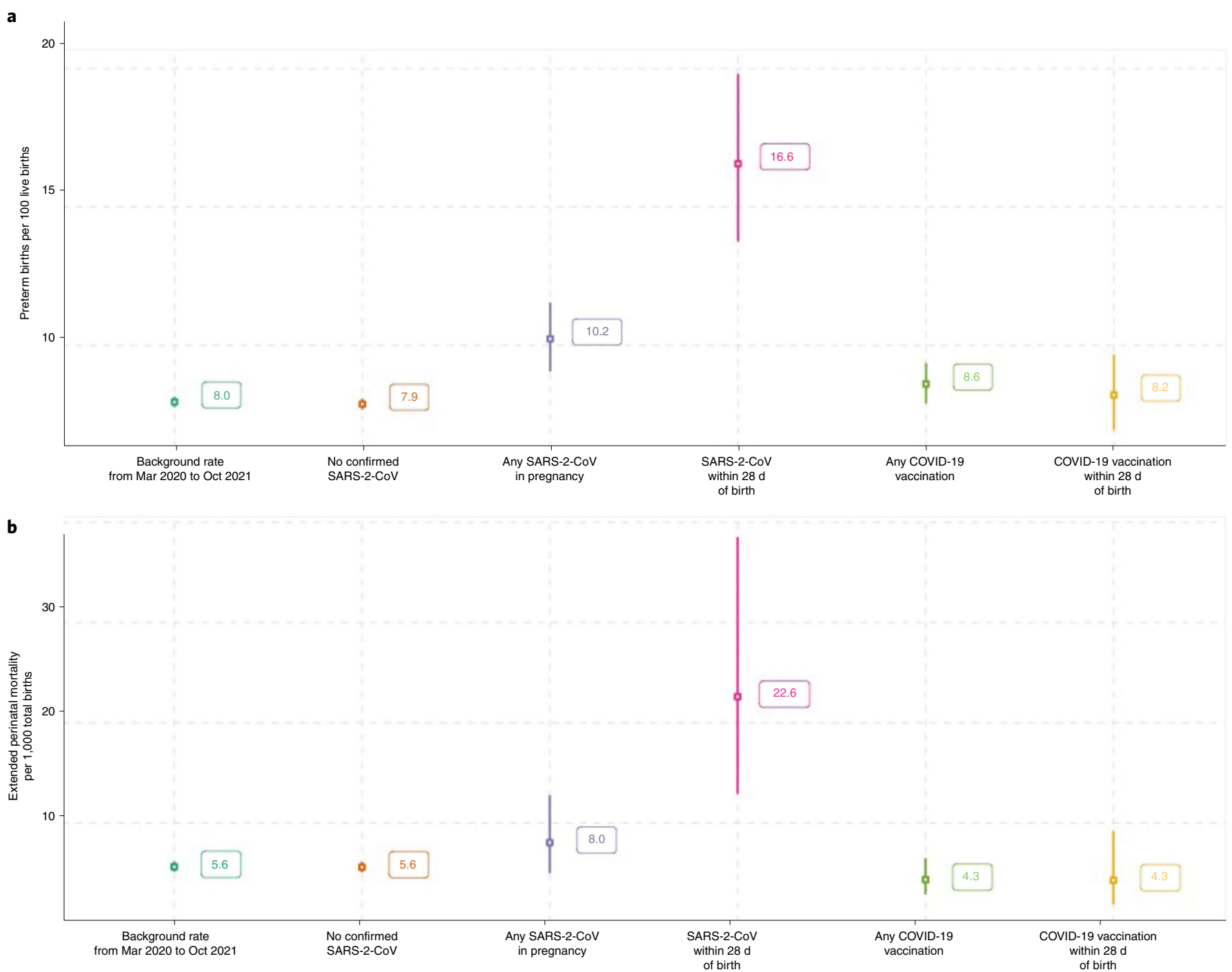

Fig. 5 | Preterm birth and perinatal mortality after SARS-CoV-2 in pregnancy. a,b, Preterm birth ( $<37$ weeks gestation) rate per 100 live births (a) and extended perinatal mortality rates (b) (stillbirths (death in utero $\geq 24$ weeks gestation) and neonatal deaths within $28 \mathrm{~d}$ of birth per 1,000 total births) in different cohorts during the pandemic. Background rate is the rate for all babies born during the pandemic period (1 March 2020 to 31 October 2021 ); no confirmed SARS-CoV-2 is the rate for babies born during the pandemic period (1 March 2020 to 31 October 2021) to women with no confirmed SARS-CoV-2 infection during pregnancy; any SARS-CoV-2 is the rate for babies born to women who had confirmed SARS-CoV-2 during their pregnancy 1 December 2020 to 31 October 2021; SARS-CoV-2 within 28 d of birth is the rate for babies born to women who had confirmed SARS-CoV-2 during their pregnancy 1 December 2020 to 31 October 2021, restricted to babies born within 28 d of the date of onset of maternal infection; Any COVID-19 vaccination is the rate for babies born to women who had COVID-19 vaccination during their pregnancy 1 December 2020 to 31 October 2021; COVID-19 vaccination within $28 \mathrm{~d}$ of birth is the rate for babies born to women who had COVID-19 vaccination during their pregnancy 1 December 2020 to 31 October 2021 , restricted to babies born within $28 \mathrm{~d}$ of the date of maternal vaccination.

births; 95\% CI 5.1-6.2). When restricted to babies born to women with no confirmed SARS-CoV-2 infection during pregnancy the preterm birth rate was $7.9 \%$ (6,083 out of 77,209 live births; $95 \%$ CI 7.7-8.1) and the extended perinatal mortality was 5.6 per 1,000 births (432 out of 77,470 total births; $95 \%$ CI $5.1-6.1$ ). These preterm and perinatal mortality rates are shown in Fig. 5, along with the preterm birth and extended perinatal mortality rates in women who received the COVID-19 vaccine in pregnancy (preterm birth rate of $8.6 \%$ (495 out of 5,752 live births in women who received COVID-19 vaccination in pregnancy; 95\% CI 7.9-9.4) and $8.2 \%$ (134 out of 1,632 live births within $28 \mathrm{~d}$ of COVID-19 vaccination in pregnancy; 95\% CI 7.0-9.6); and extended perinatal mortality of 4.3 per 1,000 births ( 25 out of 5,766 total births in women who received COVID-19 vaccination in pregnancy; 95\% CI 2.9-6.4) and
4.3 per 1,000 births ( 7 out of 1,635 births within $28 \mathrm{~d}$ of COVID-19 vaccination; 95\% CI 1.9-9.2)).

\section{Discussion}

These population data provide new national evidence of low levels of vaccine uptake in pregnancy in Scotland, at a time when the Delta variant dominated, with only $32.3 \%$ of pregnant women giving birth in October having had two doses of the vaccine. Rates were lowest in younger women and populations who lived in the most deprived areas. SARS-CoV-2 infection rates in pregnancy closely mirrored those in the general population throughout the pandemic, when different SARS-CoV-2 variants predominated in Scotland (wild type initially, Alpha dominant from January 2021 and Delta dominant from May 2021). We found that severe complications 
a

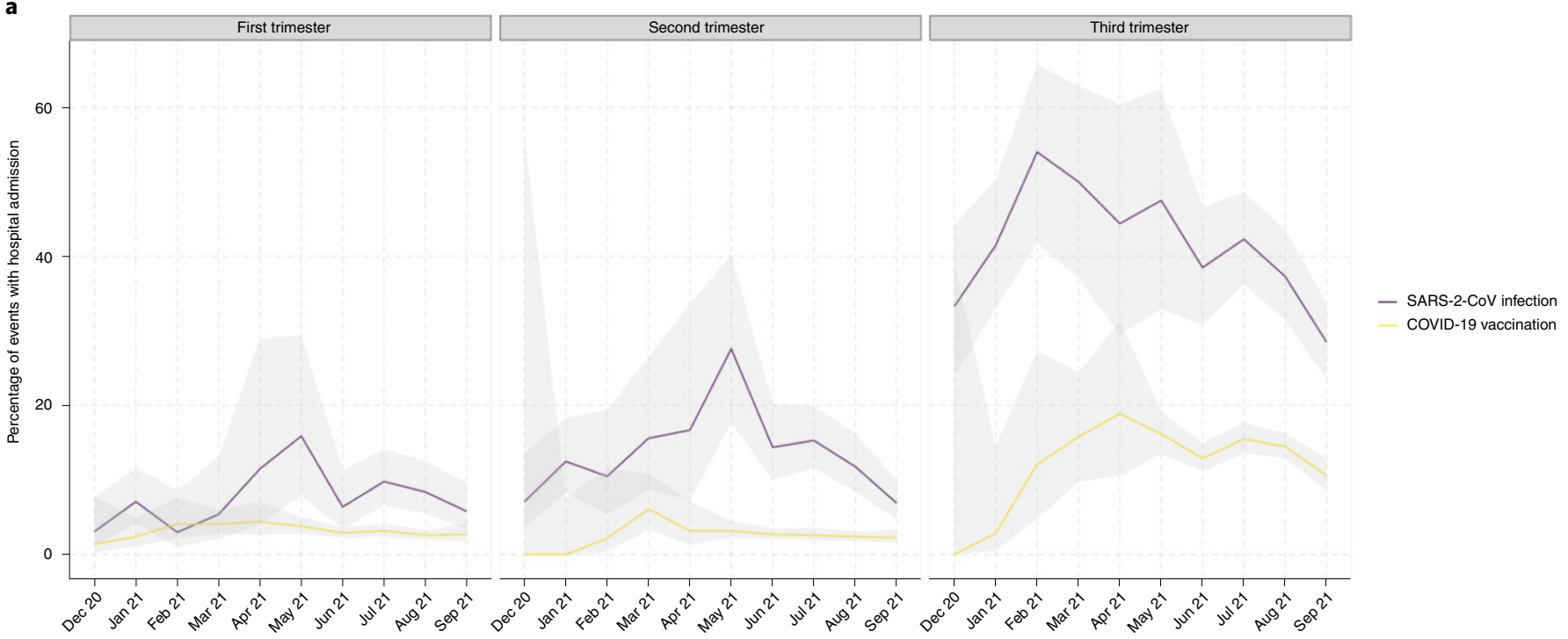

b

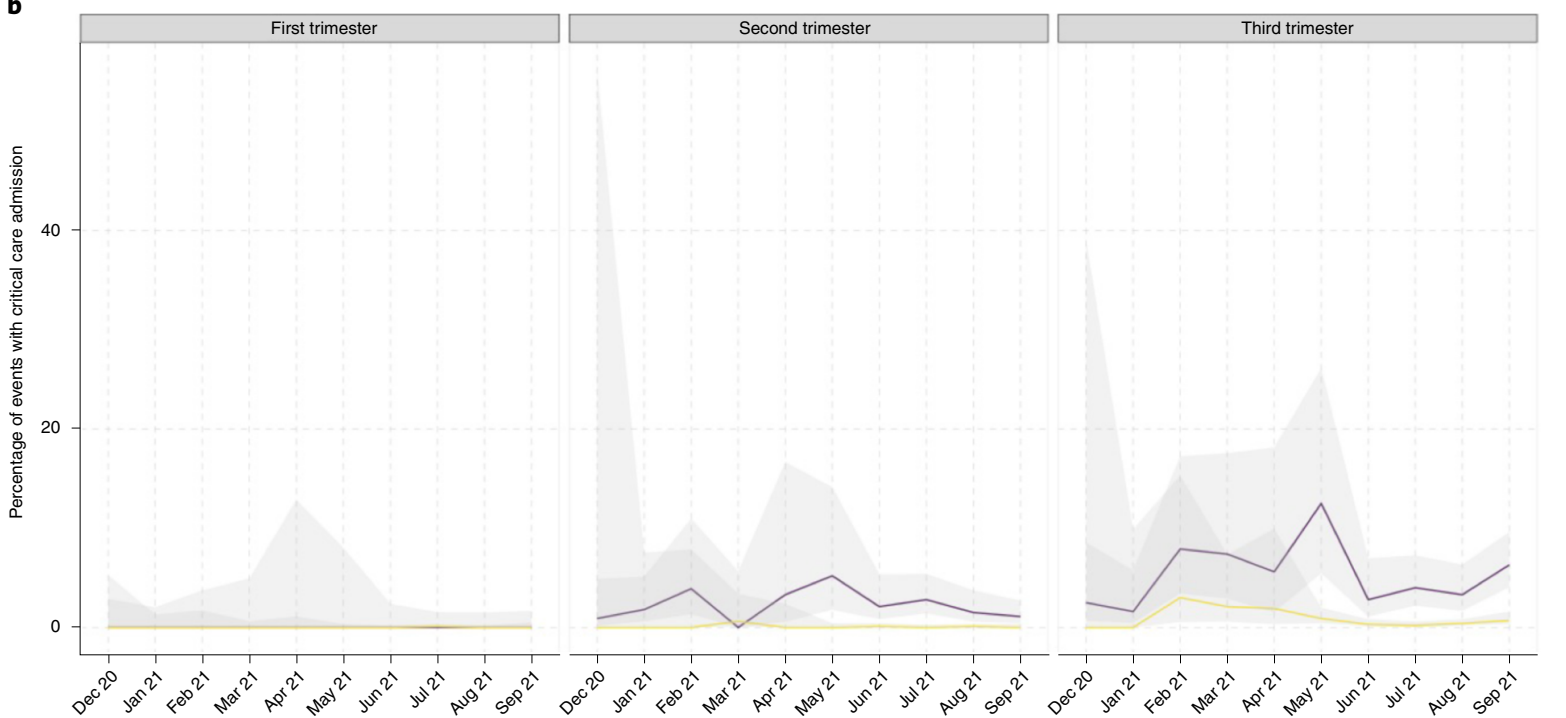

- SARS-2-CoV infection COVID-19 vaccination

Fig. 6 | Hospital and critical care admissions after SARS-CoV-2 and COVID-19 vaccination in pregnancy. a, Percentage of cases of SARS-CoV-2 infections in pregnancy associated with hospital admission (defined as hospital admission $\leq 14 \mathrm{~d}$ after the date of diagnosis of SARS-2-CoV infection or if the date of diagnosis occurred at any point during a hospital admission) and COVID-19 vaccinations in pregnancy associated with hospital admission (defined as hospital admission $\leq 14 \mathrm{~d}$ after the date of COVID-19 vaccination) occurring 1 December 2020 to 30 September 2021 . b, Percentage of SARS-CoV-2 infections in pregnancy associated with critical care admission (defined as critical care admission $\leq 21 \mathrm{~d}$ after the date of diagnosis of $\mathrm{SARS}$-CoV-2 infection or if the date of diagnosis occurred at any point during a critical care admission) and COVID-19 vaccinations in pregnancy associated with critical care admission (defined hospital admission $\leq 21 \mathrm{~d}$ after the date of COVID-19 vaccination) occurring 1 December 2020 to 30 September 2021 . Definitions are as per Public Health Scotland standards for associated hospital admission and critical care admission. Data are restricted to infections and vaccinations occurring up to 30 September 2021 due to incomplete follow-up times.

known to be associated with COVID-19 in pregnancy (critical care admission and perinatal mortality) were more common in women who were unvaccinated at the time of COVID-19 diagnosis than in vaccinated pregnant women. Our data support the importance of women being vaccinated in pregnancy to prevent adverse outcomes associated with COVID-19. For extended perinatal mortality, for example, we find substantially elevated rates among women who had SARS-CoV-2 within $28 \mathrm{~d}$ of birth; conversely, for women who had COVID-19 vaccination, extended perinatal morality rates were similar to background rates and among women with no confirmed SARS-CoV-2.

Although surveillance studies, which have mainly been based on women admitted to hospital, have suggested that pregnant women are at highest risk of severe COVID-19 disease in the third trimester ${ }^{9}$, these Scottish population data, capturing all virologically confirmed infections in a setting where testing has been widely available in hospital and the community, show that SARS-CoV-2 infections were relatively evenly spread throughout pregnancy. However, in line with findings from other studies, SARS-CoV-2 associated with hospital and critical care admission was more common as pregnancy progressed ${ }^{4,9}$.

A strength of this study is that it used population data with high data completeness. Another strength is that the data describe changes in vaccine uptake over time, with most contemporary data available included. A caveat is that due to source data latency, the most recent 3 months' pregnancy data may be less complete (there 
may be some under-ascertainment of the most recent pregnancies and end of pregnancy outcomes). A limitation of our study is that the data presented are descriptive and we have not adjusted for the potential confounding influence of demographics, obstetric or medical conditions. A fully adjusted analysis of all pregnancy outcomes (including early pregnancy outcomes) is planned ${ }^{27}$ when a greater number of end of pregnancy events have accumulated to ensure sufficient power, with appropriate historic comparison groups included ${ }^{28}$. Another limitation of our study is that we were unable to differentiate between hospital and critical care admissions due to SARS-CoV-2 infection from admissions for obstetric care with coincidental SARS-CoV-2 infection. SARS-CoV-2 infection in pregnancy may require admission in its own right for COVID-19 treatment; may trigger obstetric complications that require admission for maternity care or critical care (for example pre-eclampsia or delivery) or may be an incidental finding on admission. Due to this complexity, routine population data coding was not sufficiently detailed to identify coincidental infections. Nevertheless, comparison of hospital and critical care admissions within $28 \mathrm{~d}$ of SARS-CoV-2 infection, to those within $28 \mathrm{~d}$ of COVID-19 vaccination show different patterns (Fig. 6). Given the established safety profile of vaccinations ${ }^{18-21}$, we would anticipate that post-vaccination admissions are likely to be predominantly for obstetric indications. The higher levels of second and third trimester hospital and critical care admissions following SARS-CoV-2 infections compared to vaccinations suggests that management of COVID-19 or its obstetric sequelae is the most likely reason for excess admissions.

Our findings emphasize the need for continued efforts to increase vaccination uptake in pregnant women, especially in younger and more deprived populations ${ }^{29}$. The percentage of pregnant women vaccinated each month has declined since August 2021, reflecting the roll out of the vaccination program and the likelihood that an increasing proportion of women entering pregnancy are fully vaccinated. However, vaccine coverage, although increasing, remains low, with only a minority of pregnant women fully vaccinated by the time of delivery. Vaccine hesitancy in pregnancy thus requires addressing, especially in light of new recommendations for booster vaccination administration 3 months after the initial vaccination course to help protect against new variants such as Omicron ${ }^{30}$. Low levels of vaccine uptake in Scotland are likely to be representative of those in the United Kingdom and other high-income countries ${ }^{31}$ (Public Health Wales, personal communication). Addressing low vaccine uptake in pregnant women is imperative to protect the health of women and babies.

\section{Online content}

Any methods, additional references, Nature Research reporting summaries, source data, extended data, supplementary information, acknowledgements, peer review information; details of author contributions and competing interests; and statements of data and code availability are available at https://doi.org/10.1038/ s41591-021-01666-2.

Received: 4 November 2021; Accepted: 20 December 2021; Published online: 13 January 2022

\section{References}

1. Razzaghi, H. et al. COVID-19 vaccination coverage among pregnant women during pregnancy - eight integrated health care organizations, United States, December 14, 2020-May 8, 2021. MMWR Morb. Mortal. Wkly Rep. 70, 895-899 (2021).

2. Allotey, J. et al. Clinical manifestations, risk factors, and maternal and perinatal outcomes of coronavirus disease 2019 in pregnancy: living systematic review and meta-analysis. BMJ 370, m3320 (2020).

3. Zambrano, L. D. et al. Update: characteristics of symptomatic women of reproductive age with laboratory-confirmed SARS-CoV-2 infection by pregnancy status - United States, January 22-October 3, 2020. MMWR Morb. Mortal. Wkly Rep. 69, 1641-1647 (2020).
4. Magnus, M.C. et al. Pregnancy and risk of COVID-19: a Norwegian registry-linkage study. BJOG https://doi.org/10.1111/1471-0528.16969 (2021).

5. Villar, J. et al. Maternal and neonatal morbidity and mortality among pregnant women with and without COVID-19 infection: the INTERCOVID Multinational Cohort Study. JAMA Pediatr. 175, 817-826 (2021).

6. Papageorghiou, A. T. et al. Preeclampsia and COVID-19: results from the INTERCOVID prospective longitudinal study. Am. J. Obstet. Gynecol. 225, 289 (2021).

7. Wei, S. Q., Bilodeau-Bertrand, M., Liu, S. \& Auger, N. The impact of COVID-19 on pregnancy outcomes: a systematic review and meta-analysis. CMAJ 193, E540-E548 (2021).

8. Knight, M. et al. Characteristics and outcomes of pregnant women admitted to hospital with confirmed SARS-CoV-2 infection in UK: national population-based cohort study. BMJ 369, m2107 (2020).

9. Vousden, N. et al. The incidence, characteristics and outcomes of pregnant women hospitalized with symptomatic and asymptomatic SARS-CoV-2 infection in the UK from March to September 2020: a national cohort study using the UK Obstetric Surveillance System (UKOSS). PLoS ONE 16, e0251123 (2021)

10. Mullins, E. et al. Pregnancy and neonatal outcomes of COVID-19: coreporting of common outcomes from PAN-COVID and AAP-SONPM registries. Ultrasound Obstet. Gynecol. 57, 573-581 (2021).

11. Luxi, N. et al. COVID-19 vaccination in pregnancy, paediatrics, immunocompromised patients, and persons with history of allergy or prior SARS-CoV-2 infection: overview of current recommendations and pre- and post-marketing evidence for vaccine efficacy and safety. Drug Saf. https://doi. org/10.1007/s40264-021-01131-6 (2021).

12. Knight, M., Morris, R. K., Furniss, J. \& Chappell, L. C. Include pregnant women in research-particularly COVID-19 research. BMJ 370, m3305 (2020)

13. Johns Hopkins Berman Institute of Bioethics and the Johns Hopkins Center for Immunization Research. COVID-19 maternal immunization tracker (COMIT). https://www.comitglobal.org (2021).

14. Goldshtein, I. et al. Association between BNT162b2 vaccination and incidence of SARS-CoV-2 infection in pregnant women. JAMA 326, 728-735 (2021)

15. Gray, K. J. et al. Coronavirus disease 2019 vaccine response in pregnant and lactating women: a cohort study. Am. J. Obstet. Gynecol. 225, 303 (2021).

16. Center for Disease Control (CDC). If you are pregnant, breastfeeding, or caring for young children. https://www.cdc.gov/coronavirus/2019-ncov/ need-extra-precautions/pregnancy-breastfeeding.html?CDC_AA_ refVal=https\%3A\%2F\%2Fwww.cdc.gov\%2Fcoronavirus\%2F2019-ncov\%2Fhcp \%2Fpregnant-women-faq.html (2020).

17. Hillson, K. et al. Fertility rates and birth outcomes after ChAdOx1 nCoV-19 (AZD1222) vaccination. Lancet 398, 1683-1684 (2021).

18. Shimabukuro, T. T. et al. Preliminary findings of mRNA COVID-19 vaccine safety in pregnant persons. N. Engl. J. Med. 384, 2273-2282 (2021).

19. Wainstock, T., Yoles, I., Sergienko, R. \& Sheiner, E. Prenatal maternal COVID-19 vaccination and pregnancy outcomes. Vaccine 39, 6037-6040 (2021)

20. Theiler, R. N. et al. Pregnancy and birth outcomes after SARS-CoV-2 vaccination in pregnancy. Am. J. Obstet. Gynecol. MFM 3, 100467 (2021).

21. Zauche, L. H. et al. Receipt of mRNA COVID-19 vaccines and risk of spontaneous abortion. N. Engl. J. Med. 385, 1533-1535 (2021).

22. Simpson, C.R. et al. Early pandemic evaluation and enhanced surveillance of COVID-19 (EAVE II): protocol for an observational study using linked Scottish national data. BMJ Open 10, e039097 (2020).

23. Mulholland, R. H. et al. Cohort profile: early pandemic evaluation and enhanced surveillance of COVID-19 (EAVE II) database. Int. J. Epidemiol. 50, 1064-1074 (2021)

24. Stock, S. J. et al. COVID-19 in pregnancy in Scotland (COPS): protocol for an observational study using linked Scottish national data. BMJ Open 10, e042813 (2020).

25. Stock S. J. et al. Cohort profile: the COVID-19 in pregnancy in Scotland (COPS) dynamic cohort of pregnant women to assess effects of viral and vaccine exposures on pregnancy. Int. J. Epidemiol. https://doi.org/10.1093/ije/ dyab243 (2022)

26. Public Health Scotland. Open data. https://www.opendata.nhs.scot/dataset/ covid-19-in-scotland (2021).

27. Public Health Scotland. COPS-public Git Hub. https://github.com/ Public-Health-Scotland/COPS-public (2021).

28. Knight, M., Draper, E. \& Kurinczuk, J. J. Misclassification bias and unnecessary anxiety. Am. J. Obstet. Gynecol. 225, 584 (2021).

29. Iacobucci, G. COVID-19 and pregnancy: vaccine hesitancy and how to overcome it. BMJ 375, n2862 (2021)

30. The Joint Committee on Vaccination and Immunisation (JCVI) advice on the UK vaccine response to the Omicron variant. https://www.gov.uk/ government/publications/ uk-vaccine-response-to-the-omicron-variant-jcvi-advice/ jcvi-advice-on-the-uk-vaccine-response-to-the-omicron-variant (2021). 
31. UK Health Security Agency. Vaccine surveillance report. https://assets. publishing.service.gov.uk/government/uploads/system/uploads/attachment_ data/file/1036047/Vaccine_surveillance_report_-_week_47.pdf (2021).

Publisher's note Springer Nature remains neutral with regard to jurisdictional claims in published maps and institutional affiliations.

(c) Open Access This article is licensed under a Creative Commons Attribution 4.0 International License, which permits use, sharing, adaptation, distribution and reproduction in any medium or format, as long as you give appropriate credit to the original author(s) and the source, provide a link to the Creative Commons license, and indicate if changes were made. The images or other third party material in this article are included in the article's Creative Commons license, unless indicated otherwise in a credit line to the material. If material is not included in the article's Creative Commons license and your intended use is not permitted by statutory regulation or exceeds the permitted use, you will need to obtain permission directly from the copyright holder. To view a copy of this license, visit http://creativecommons. org/licenses/by/4.0/.

(C) The Author(s) 2022, corrected publication 2022 


\section{Methods}

Ethics and data governance. COPS is a sub-study of EAVE II, using unconsented data that is covered by National Research Ethics Service Committee, South East Scotland 02 approval reference REC 12/SS/0201: SA 2. COPS has been approved by the Public Benefit and Privacy Panel approval reference 2021-0116. Public Health Scotland and the Chief Medical Officer for Scotland are both (independent) data controllers for the national Abortion Act Scotland (AAS) database of termination of pregnancy notifications, thus the Chief Medical Officer has been informed of the use of AAS records. All data were housed within a secure trusted research environment within Public Health Scotland and accessed only by approved researchers.

Study design and population. COPS is a prospective dynamic pregnancy cohort, identifying all ongoing and completed pregnancies in Scotland, United Kingdom, since 1 March 2020 (ref. ${ }^{24}$ ). In this manuscript we used the COPS database as updated in mid-November 2021 linked to records of COVID-19 vaccinations delivered and SARS-CoV-2 infections diagnosed up to and including 31 October 2021. Further detail on the datasets used are in the published protocol ${ }^{24}$. The COPS data dictionary is available online ${ }^{28}$.

Maternity data sources and linkage. Ongoing pregnancies were identified using antenatal booking appointment data. Completed pregnancies were identified using multiple sources, specifically general practitioner records, general acute hospital discharge records, Scottish Morbidity Record (SMR) 01; maternity hospital discharge records, SMR 02; notification of termination data as governed by AAS; National Records of Scotland (NRS) statutory live birth registrations; NHS Scotland live births; and NRS statutory stillbirth registrations. These datasets capture miscarriage, molar pregnancy or ectopic pregnancy requiring any general practitioner or hospital-based care, a termination of pregnancy, a live birth or a stillbirth.

The CHI number is a unique patient identifier used across all health records in Scotland, enabling integration of healthcare data. All source data incorporated into COPS were subject to a CHI-seeding process in which reported CHIs are reconciled to a contemporaneous unique patient identifier to ensure accurate linkage across sources.

Where multiple records within a data source pertained to the same woman, records were subject to event resolution, in which an iterative grouping procedure assigned records occurring within $83 \mathrm{~d}$ (less than 12 weeks) to a single healthcare event with its own universally unique identifier. The estimated date of conception was used to resolve records to a single pregnancy with a single universally unique identifier when gestation information was available and multiple records within a data source pertained to the same woman. Following event resolution, records from each individual data source were linked together to create a comprehensive record for each pregnancy and birth.

The COPS cohort is updated monthly and although source data latency means there is some instability in data for the most recent months, data are generally complete for conceptions and end of pregnancy events occurring up to 3 months previously.

National resources for SARS-CoV-2 infection data and COVID-19 vaccination data were incorporated into the study using $\mathrm{CHI}$ linkage.

Statistical Analysis. Results are based on simple descriptive statistics, for example calculation of the proportion of vaccination events occurring by trimester, or the extended perinatal mortality rate following maternal infection, with relevant definitions provided. Wilson score estimates were used for $95 \%$ confidence intervals. All analyses were performed in R version 3.6.1.

Infection and vaccination in pregnancy was defined as infection diagnosed or vaccination given, at any point from the date of conception $(2+0$ weeks gestation) to the date the pregnancy ends inclusive (censoring infections and vaccinations occurring at $44+0$ weeks gestation or over as it is very likely that these women have completed their pregnancy, but the end of pregnancy record has not yet been received by Public Health Scotland). The date of first positive viral RT-PCR sample collection was taken as the date of onset of the first episode of COVID-19. Subsequent episodes were recorded if a positive viral RT-PCR sample was taken $\geq 90 \mathrm{~d}$ after a first positive sample. Lateral flow test results were not considered. For the duration of our study period, anyone in Scotland having a positive lateral flow test was advised to have a follow up RT-PCR test to confirm COVID-19.

Vaccination status was defined as unvaccinated (no previous COVID-19 vaccination before the date of onset of COVID-19 or with one dose of vaccination $\leq 21 \mathrm{~d}$ before the date of onset), partially vaccinated (one dose of vaccination $>21 \mathrm{~d}$ before the date of onset of COVID-19 or two doses of vaccination with the second dose $\leq 14 \mathrm{~d}$ before the date of onset) or fully vaccinated (two doses of vaccination with the second dose $>14 \mathrm{~d}$ before the date of onset of COVID-19).

Monthly vaccine uptake was calculated as the number of pregnant women vaccinated during the month divided by the number of women with ongoing pregnancies at the start of the month of interest. Coverage of vaccination at delivery, was calculated as the proportion of women who, at the time of giving birth, have been vaccinated, whether vaccination was before or during pregnancy. Uptake in the general female population of reproductive age (18-44 years) was calculated as the number of women 18-44 years of age vaccinated during the month divided by the total number of women 18-44 years of age. Coverage in the general female population of reproductive age (18-44 years) was calculated as the cumulative number of women 18-44 years of age vaccinated by the end of each month divided by the total number of women $18-44$ years of age. Numbers of women 18-44 years were derived from 2020 mid-year population estimates ${ }^{26}$. Although pregnant women 11-55 years of age were included in the COPS cohort, the comparator group was restricted to women $18-44$ years. This is the Public Health Scotland standard definition of women of reproductive age and helps avoid bias in comparisons that could result from very low numbers of pregnancies at the extremes of reproductive age.

National hospital discharge records were linked to pregnancy SARS-CoV-2 RT-PCR testing data to identify women who were admitted to hospital around the time of infection. Admissions to general acute units (SMR 01) and maternity units (SMR 02) were included. SARS-CoV-2 infection was defined as associated with a hospital admission if the woman was admitted to hospital $\leq 14 \mathrm{~d}$ after the date of onset of COVID-19 or if the date of onset occurred at any point during a hospital admission (as per Public Health Scotland standard definition). National critical care discharge records from the Scottish Intensive Care Society Audit Group were used to identify women who were admitted to critical care. Completed admissions to all intensive care units and general (non-obstetric) high-dependency units across Scotland were included. Completed admissions to the seven obstetric high-dependency units that contribute data to Scottish Intensive Care Society Audit Group (collectively covering around $60 \%$ of deliveries in Scotland) were also included. SARS-CoV-2 infection was defined as associated with a critical care admission if the woman was admitted to critical care $\leq 21 \mathrm{~d}$ after the date of onset of COVID-19 or if the date of onset occurred at any point during a critical care admission (as per Public Health Scotland standard definition).

Comparator data. Comparator data from the general population of women of reproductive age (taken as 18-44 years for these analyses) were obtained from Public Health Scotland Open data with mid-year population estimate denominators $(2020)^{26}$

Data quality and completeness. The national Scottish data used within this study undergo regular assessment and quality assurance with high level of completeness and accuracy ${ }^{32,33}$. The CHI number was used to link the SARS-CoV-2 PCR testing data and COVID-19 vaccination records to the pregnancy records. Quality of linkage was assured by the testing, vaccination and pregnancy records including complete and accurate information on individuals' $\mathrm{CHI}$ number, as confirmed in the Results section.

Reporting Summary. Further information on research design is available in the Nature Research Reporting Summary linked to this article.

\section{Data availability}

Aggregate data files of infections among pregnant women and the general population and vaccinations delivered to pregnant women and the general population are available here: https://www.opendata.nhs.scot/organization/health protection. Patient-level data underlying this article cannot be shared publicly due to data protection and confidentiality requirements. Public Health Scotland and the Chief Medical Officer for Scotland are the data holders for the data used in this study. Data can be made available to approved researchers for analysis after securing relevant permissions from the data holders via the Public Benefit and Privacy Panel. Enquiries regarding data availability should be directed to phs.edris@phs.scot.

\section{Code availability}

Metadata and code are available at https://github.com/Public-Health-Scotland/ COPS-public.

\section{References}

32. NSS Information and Intelligence Assessment of SMR02 (Maternity Inpatient and Day Case) Data; Scotland 2017-2018. https://www.isdscotland.org/ Products-and-Services/Data-Quality/docs/20191023-Assessmentof-SMR02-Data-Scotland-2017-2018.pdf (2019).

33. Public Health Scotland. Data Quality Assurance Assessment of SMR01 (Acute Inpatient and Day Case) Data Scotland 2019-2020. https://www.isdscotland. org/Health-Topics/Hospital-Care/Publications/2012-05-08/ Assessment-of-SMR01Data-2010-2011-ScotlandReport.pdf (2020).

\section{Acknowledgements}

We thank the EAVE II Patient Advisory Group and Sands charity for their support. COPS is a sub-study of EAVE II, which is funded by the Medical Research Council (MR/R008345/1) with the support of BREATHE, the Health Data Research Hub for Respiratory Health (MC_PC_19004; A.S.), which is funded through the UK Research and Innovation Industrial Strategy Challenge Fund and delivered through Health Data Research UK. Additional support has been provided through Public Health Scotland and Scottish Government DG Health and Social Care and the Data and Connectivity National Core Study, led by Health Data Research UK in partnership with the Office 
for National Statistics and funded by UK Research and Innovation. COPS has received additional funding from Tommy's charity and support from Sands charity. S.J.S. is funded by a Wellcome Trust Clinical Career Development Fellowship (209560/Z/17/Z) S.V.K. acknowledges funding from an NRS Senior Clinical Fellowship (SCAF/15/02), the Medical Research Council (MC_UU_00022/2) and the Scottish Government Chief Scientist Office (SPHSU17). The funders had no role in the writing of the manuscript or the decision to submit it for publication. B.A. was supported by the European Union's Horizon 2020 research and innovation programme under the Marie Skłodowska-Curie grant agreement No. 813546, the Baily Thomas Charitable Fund TRUST/VC/AC/ SG/469207686, the Data Driven Innovation and the UK Economic and Social Research Council (ES/W001519/1) during the course of this work.

\section{Author contributions}

S.J.S., R.W., C.R. and A.S. conceived the study. S.J.S., E.V., C.R.S., U.A., C.M., J.D., L.R., C.R., A.S., L.H., L.E.M.H., A.G., J.C., T.M., J.P., C.C. and R.W. designed the study. S.J.S., E.V., C.R.S., U.A., C.M., L.H., J.D., L.R., C.R., A.S., R.M., S.V.K. and R.W. drafted the protocol. J.D., A.G., C.D., L.H., L.E.M.H., T.M. and J.C. performed data analysis. S.J.S., E.V., C.R.S., U.A., C.M., L.H., L.R., C.R., A.S., A.G., C.D., L.E.M.H., J.C., B.A., C.C., J.P., J.M. and R.W. interpreted data and revised the manuscript for important intellectual content. S.J.S., D.M., E.V., C.R.S., U.A., C.M., L.H., J.D., L.R., C.R., A.S., A.G., C.D., L.E.M.H., J.C., B.A., S.V.K., C.C., J.P., J.M. and R.W. gave final approval of the version to be published. R.W. acts as guarantor for the study.

\section{Competing interests}

A.S. and C.R. are members of the Scottish Government's COVID-19 Advisory Group. A.S. and C.R. are members of the New and Emerging Respiratory Virus Threats Advisory Group risk stratification subgroup. C.R. is a member of the Scientific Pandemic Influenza Group on Modeling. A.S. is a member of AstraZeneca's Thrombotic Thrombocytopenic Advisory Group. All roles are unremunerated. R.W. and C.R. are employed by Public Health Scotland. S.J.S. has received research grants paid to the institution from Wellcome Trust, Scottish Chief Scientist Office, National Institute of Healthcare Research and Tommy's during the course of this study. The remaining authors declare no competing interests.

\section{Additional information}

Supplementary information The online version contains supplementary material available at https://doi.org/10.1038/s41591-021-01666-2.

Correspondence and requests for materials should be addressed to Sarah J. Stock.

Peer review information Nature Medicine thanks Gianluca Trifirò, Deborah Money and Denise Jamieson for their contribution to the peer review of this work. Jennifer Sargent was the primary editor on this article and managed its editorial process and peer review in collaboration with the rest of the editorial team.

Reprints and permissions information is available at www.nature.com/reprints. 


\section{Reporting Summary}

Nature Portfolio wishes to improve the reproducibility of the work that we publish. This form provides structure for consistency and transparency in reporting. For further information on Nature Portfolio policies, see our Editorial Policies and the Editorial Policy Checklist.

\section{Statistics}

For all statistical analyses, confirm that the following items are present in the figure legend, table legend, main text, or Methods section.

$\mathrm{n} / \mathrm{a} \mid$ Confirmed

$\bigotimes$ The exact sample size $(n)$ for each experimental group/condition, given as a discrete number and unit of measurement

Х $\square$ A statement on whether measurements were taken from distinct samples or whether the same sample was measured repeatedly

Х The statistical test(s) used AND whether they are one- or two-sided

Xnly common tests should be described solely by name; describe more complex techniques in the Methods section.

Х $\square$ A description of all covariates tested

Х $\square$ A description of any assumptions or corrections, such as tests of normality and adjustment for multiple comparisons

$\varnothing$ A full description of the statistical parameters including central tendency (e.g. means) or other basic estimates (e.g. regression coefficient)

X AND variation (e.g. standard deviation) or associated estimates of uncertainty (e.g. confidence intervals)

For null hypothesis testing, the test statistic (e.g. $F, t, r$ ) with confidence intervals, effect sizes, degrees of freedom and $P$ value noted

$\square$ Give $P$ values as exact values whenever suitable.

Х $\square$ For Bayesian analysis, information on the choice of priors and Markov chain Monte Carlo settings

$\bigotimes \square$ For hierarchical and complex designs, identification of the appropriate level for tests and full reporting of outcomes

$\bigotimes \square$ Estimates of effect sizes (e.g. Cohen's $d$, Pearson's $r$ ), indicating how they were calculated

Our web collection on statistics for biologists contains articles on many of the points above.

\section{Software and code}

Policy information about availability of computer code

Data collection R version 3.6.1

Data analysis R version 3.6.1

For manuscripts utilizing custom algorithms or software that are central to the research but not yet described in published literature, software must be made available to editors and

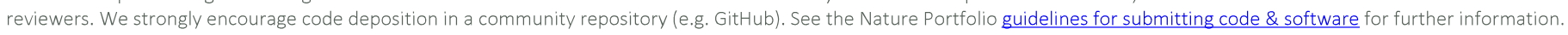

\section{Data}

Policy information about availability of data

All manuscripts must include a data availability statement. This statement should provide the following information, where applicable:

- Accession codes, unique identifiers, or web links for publicly available datasets

- A description of any restrictions on data availability

- For clinical datasets or third party data, please ensure that the statement adheres to our policy

Data files of infections among pregnant women and the general population and vaccinations delivered to pregnant women and the general population are available here: https://www.opendata.nhs.scot/organization/health_protection.

Other personal data underlying this article cannot be shared publicly as they are sensitive. Public Health Scotland and the Chief Medical Officer for Scotland are the data holders for the data used in this study. Data are available to researchers for analysis after securing relevant permissions from the data holders. Enquiries regarding data availability should be directed to phs.edris@phs.scot. 
Please select the one below that is the best fit for your research. If you are not sure, read the appropriate sections before making your selection.

$\bigotimes$ Life sciences $\quad \square$ Behavioural \& social sciences $\quad \square$ Ecological, evolutionary \& environmental sciences

For a reference copy of the document with all sections, see nature.com/documents/nr-reporting-summary-flat.pdf

\section{Life sciences study design}

All studies must disclose on these points even when the disclosure is negative.

Sample size
Data exclusions is a descriptive study using routinely collected health data. No sample size calculation was performed.
$\begin{aligned} & \text { Data were excluded if there were pre-specified unfeasible values, as described in the data dictionary available from https://github.com/Public- } \\ & \text { Health-Scotland/COPS-public. }\end{aligned}$
Replication N/A - descriptive study of routinely collected health data.
Randomization N/A - descriptive study of routinely collected health data.
Blinding N/A - descriptive study of routinely collected health data.

\section{Reporting for specific materials, systems and methods}

We require information from authors about some types of materials, experimental systems and methods used in many studies. Here, indicate whether each material, system or method listed is relevant to your study. If you are not sure if a list item applies to your research, read the appropriate section before selecting a response.

\begin{tabular}{|c|c|}
\hline $\mathrm{n} / \mathrm{a}$ & Involved in the study \\
\hline Х & $\square$ Antibodies \\
\hline Х & $\square$ Eukaryotic cell lines \\
\hline Х & $\square$ Palaeontology and archaeology \\
\hline Х & $\square$ Animals and other organisms \\
\hline - & $\bigotimes$ Human research participants \\
\hline Х & $\square$ Clinical data \\
\hline Х & $\square$ Dual use research of concern \\
\hline
\end{tabular}

\begin{tabular}{l|l}
\multicolumn{2}{l}{ Methods } \\
\hline n/a & Involved in the study \\
$\searrow$ & $\square$ ChIP-seq \\
$\searrow$ & $\square$ Flow cytometry \\
$\searrow$ & $\square$ MRI-based neuroimaging
\end{tabular}

\section{Human research participants}

Policy information about studies involving human research participants

Population characteristics

Recruitment

Ethics oversight
Women (15 to 55 years) who had early pregnancy loss, termination of pregnancy, live or stillbirth, neonatal death, or antenatal care for an ongoing pregnancy.

National, prospective dynamic cohort of routinely collected healthcare data.

COPS is a sub-study of EAVE II, using unconsented data, which is covered by National Research Ethics Service Committee, South East Scotland 02 approval reference REC 12/SS/0201: SA 2. COPS has been approved by the Public Benefit and Privacy Panel approval reference 2021-0116. Public Health Scotland and the Chief Medical Officer for Scotland are both (independent) data controllers for the national Abortion Act Scotland (AAS) database of termination of pregnancy notifications, thus the Chief Medical Officer has been informed of the use of AAS records for this study. 